\title{
Prognostic role of long non-coding RNA XIST expression in patients with solid tumors: a meta-analysis
}

\author{
Huihui Mao ${ }^{1}$, Kai Wang ${ }^{2}$, Yuehua Feng ${ }^{1}$, Jun Zhang ${ }^{1}$, Lili Pan ${ }^{1}$, Yuxia Zhan ${ }^{1}$, Haijun Sheng ${ }^{1}$ and Guanghua Luo ${ }^{1 *}$
}

\begin{abstract}
Background: The aberrant expression of long non-coding RNA (IncRNA) X inactivate-specific transcript (XIST) has been demonstrated to be involved in the tumourigenesis and the development of various cancers. Therefore, we conducted a meta-analysis to assess the prognostic role of IncRNA XIST expression in solid tumors.

Methods: The databases of PubMed, EMBase, Web of Science, Cochrane library (up to Dec 31, 2017) were searched for the related studies and identified 15 eligible studies containing 1209 patients to include in the meta-analysis. Hazards ratios (HRs) with corresponding 95\% confidence intervals (Cls) were pooled to estimate the association between IncRNA XIST expression and survival of cancer patients from Asian.
\end{abstract}

Results: The result showed that higher IncRNA XIST expression in cancer tissue was related to a worse overall survival (OS) ( $\mathrm{HR}=1.54,95 \% \mathrm{Cl} 1.07-2.23)$. In subgroup analysis, it revealed that IncRNA XIST overexpression was significantly associated with worse OS in digestive system tumors ( $\mathrm{HR}=1.67,95 \% \mathrm{Cl} 1.11-2.51, p=0.031)$. In addition, the association between high IncRNA XIST expression and poor OS was also statistically significant in other subgroups, including multivariate analysis ( $\mathrm{HR}=2.39,95 \% \mathrm{Cl} 1.28-4.46, p=0.006$, random-effect), patients' number was greater than 65 $(H R=1.75,95 \% \mathrm{Cl} 1.24-2.47, p=0.001$, random-effect), and reported in text $(\mathrm{HR}=2.50,95 \% \mathrm{Cl} 1.49-4.18, p=0.000$, random-effect).

Conclusions: The expression of IncRNA XIST could be regarded as a poor prognostic biomarker for solid tumors, which might shed new light on epigenetic diagnostics and therapeutics in tumors.

Keywords: Long non-coding RNA, XIST, Prognosis, Meta-analysis

\section{Background}

Both the number of cancer patients and the mortality rate are disturbingly increasing. Cancer has become a common disease that is seriously detrimental to human health, which is a significant cause of death in many countries around the world. Despite the dramatic developments in the diagnosis and therapy of tumors over the past few decades, the overall survival (OS) of patients remains unsatisfactory. Tumor markers play a significant role in monitoring and treating tumors. However, fewer

\footnotetext{
*Correspondence: shineroar@163.com

${ }^{1}$ Comprehensive Laboratory, Changzhou Key Lab of Individualized Diagnosis and Treatment Associated with High Technology Research, The Third Affiliated Hospital of Soochow University, Changzhou 213003, China Full list of author information is available at the end of the article
}

tumor markers were used in clinical diagnosis. Therefore, it is urgent need to discover molecular biomarkers to improve the sensitivity and specificity for the detection and prognosis for cancer.

With the development of high-throughput sequencing technology, an increasing number of long non-coding RNAs (lncRNAs) have been gradually discovered and become the hotspot of research. LncRNAs, which cannot encode proteins, are important members of the noncoding RNA family. The biological functions of lncRNAs are still in its infancy and no definitive conclusion has been reached on its function and clinical significance of lncRNA. Recently, accumulating evidences have indicated that lncRNAs were closely related to initiation and progression of human diseases, especially cancer. 
LncRNAs could be used as a carcinogen or suppressor gene in the development and progression of cancer.

$\mathrm{X}$-inactive specific transcript (XIST) is a kind of lncRNA derived from XIST gene that only expressed from the inactive $X$ chromosome [1,2]. Many clinical studies have clarified that the expressions of lncRNA XIST not only played an important role in the differentiation, proliferation, and genome maintenance of cells, but also with the development and progression of cancer [3]. For instance, the perturbation of IncRNA XIST expression related to metastasis and recurrence in a variety of cancers, including bladder cancer [4], nasopharyngeal carcinoma (NPC) [5], pancreatic cancer (PC) [6], colorectal cancer (CRC) [7-9], glioma [10, 11], prostate cancer (PCa) [12], ovarian cancer, gastric cancer (GC) [13, 14], hepatocellular carcinoma (HCC) $[15,16]$, and nonsmall cell lung cancer (NSCLC) [17, 18]. Nevertheless, the consistency and magnitude of the prognostic impact of lncRNA XIST remains enigmatic, and the prognostic value of lncRNA XIST expression in different tumor types remains still controversial. To verify its clinical relevance, we integrated all published evidence systematically in this meta-analysis to reveal the prognostic value of lncRNA XIST in various types of solid tumors.

\section{Materials and methods}

\section{Search strategy}

A systematic review of the literature was conducted according to the PRISMA guidelines. PubMed, EMBase, Web of Science, Cochrane library were searched to evaluate the impact of IncRNA XIST expression on survival in solid tumors. The following search terms included: "Long non coding RNA XIST" OR "Long Noncoding RNA XIST" OR "long non-coding RNA XIST" OR "lncRNA XIST" OR "X-inactive specific transcript" (all fields) AND "Prognosis" OR "Prognoses" OR "Prognostic" OR "Outcome" OR "survival" (all fields) AND "Neoplasia" OR "Neoplasias" OR "Neoplasm" OR "Tumor" OR "Cancer" OR "tumour" OR "carcinoma" (all fields). Moreover, the literature has been tracked to determine more relevant studies.

\section{Selection criteria}

All collected studies were included in this meta-analysis according to the criteria as follows: (1) lncRNAncRNA XIST expression was detected only in solid tumors, not including hematologic malignancies; (2) investigation of the association between lncRNA XIST expression and survival outcome were represented in overall survival; (3) reporting sufficient data to estimate the hazard ratio (HR) and 95\% confidence interval (CI) according to lncRNA XIST expression; (4) lncRNA XIST expression was detected by quantitative reverse transcription PCR
(qRT-PCR) in OS tissues; (5) not a review, meta-analysis, case reports, duplicate publications.

\section{Data extraction and quality assessment}

Data extraction of literature was as follows: first author, publication year, country of origin, cancer type, sample size, number of patients in high and low lncRNA XIST expression groups, the detection method, and the cut-off, survival analysis, the HR and $95 \%$ CI. If HR was provided in the study, we extracted them directly. Otherwise, survival data were extracted from the original study data (Kaplan-Meier curves or required data) using the software Engauge Digitizer 4.1 and calculated by Tierney. The quality of included studies was evaluated by two investigators independently according to the Newcastle-Ottawa Quality Assessment Scale (NOS). Furthermore, two investigators could resolve their differences by consensus or in discussions with a third investigator. The lowest and highest scores were scored at 0 and 9 , respectively, and a study with a score greater than 6 or higher was considered a high-quality study.

\section{Statistical analysis}

HR with 95\% CI was estimated to evaluate the effective value of lncRNA XIST expression on prognosis in solid tumors. The high expression and low expression of lncRNA XIST was defined according to the cut-off values provided in the article. The heterogeneity of pooled results was evaluated using Cochran's $Q$ test and Higgins I-squared statistic. A statistically significant heterogeneity was defined as $p<0.10$ or $\mathrm{I}^{2}>50 \%$, where a randomeffect was applied. Otherwise a fixed-effect model was used. Subgroup analysis was used to further explore possible sources of heterogeneity. The stability of the results was assessed using a sensitivity analysis. The possibility of publication bias was also assessed using Begg's test. All data were analyzed using STATA software version 12.0 (Stata Corporation, College Station, TX, USA), and a $p$ value less than 0.05 was considered as statistically significant.

\section{Results \\ Study characteristics}

A total of 171 related articles were retrieved, of which 169 articles were initially searched according to the criteria described in "Materials and methods" and the other 2 articles were obtained by searching the references. After screening the titles, abstracts, publication types and full text, 25 articles investigated the correlation between lncRNA XIST expression and patient survival in various tumors were selected for the systemic review. Among these, 10 articles were excluded (nine lacked some important data and one detected lncRNA XIST not in tissue 
sample) (Fig. 1). The total number of patients was 1209 patients from China and Japan to include in the metaanalysis, ranging from 41 to 145 patients (all considered research refers to the Asian population). The category of cancers included GC, NPC, NSCLC, HCC, cervical squamous cell carcinoma (CSCC), esophageal squamous cell carcinoma (ESCC), bladder cancer, PC, CRC, glioma, $\mathrm{PCa}$, and osteosarcoma. The expression levels of lncRNA XIST was detected using qRT-PCR in all studies. OS was reported in 15 studies, while disease-free survival (DFS) had only one study. Therefore, we selected OS as the main survival outcome of all eligible studies for our meta-analysis. HR was reported directly in 6 studies and estimated indirectly in the other 9 studies. The cut-off estimates for lncRNA XIST expression was different in these studies, including the mean, median, or fold change. The detailed information about the studies was shown in Table 1.

\section{Quality assessment}

According to the NOS, each of the 15 eligible studies included in our meta-analysis was assessed for quality. Each one got a higher value, which indicated a better methodology. Therefore, all 15 studies were included in the subsequent analysis.

\section{Meta-analysis results}

The main results of the meta-analysis were shown in Table 2 . The heterogeneity of 15 studies was statistically significant $\left(\mathrm{I}^{2}=86.3 \%, p<0.001\right)$, and the random-effects model was used to calculate the pooled HR and its $95 \%$ CI, which was significantly different $(\mathrm{HR}=1.54,95 \%$ CI 1.07-2.23, $p=0.021$ ). The result showed that higher IncRNA XIST expression in cancer tissue was related to a worse OS (Fig. 2).

Subsequently, subgroup analysis was performed to further explore the sources of heterogeneity among these studies based on four main characteristics including
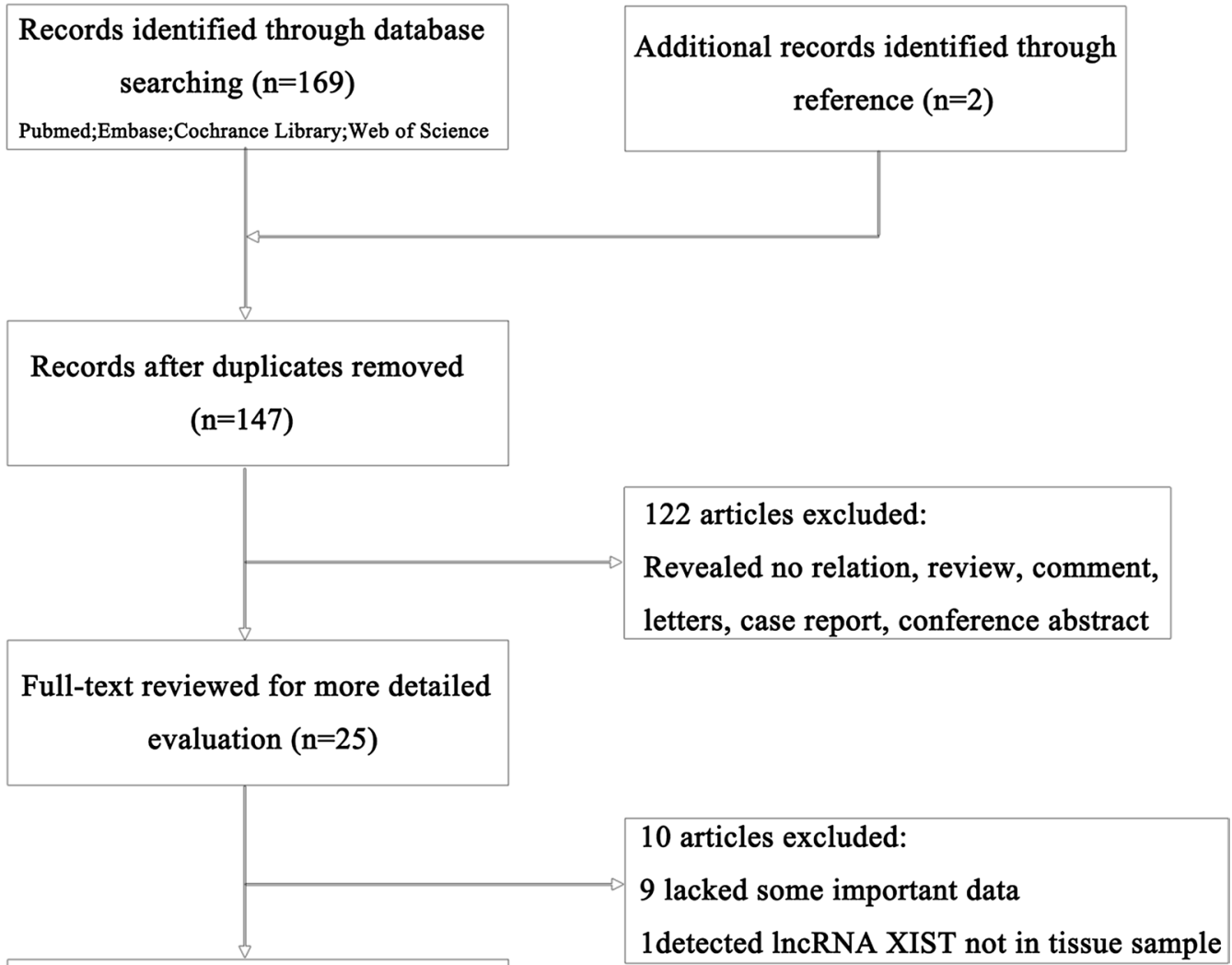

Studies eligible for inclusion in meta-analysis $(n=15)$

Fig. 1 Flow diagram of the study selection process 


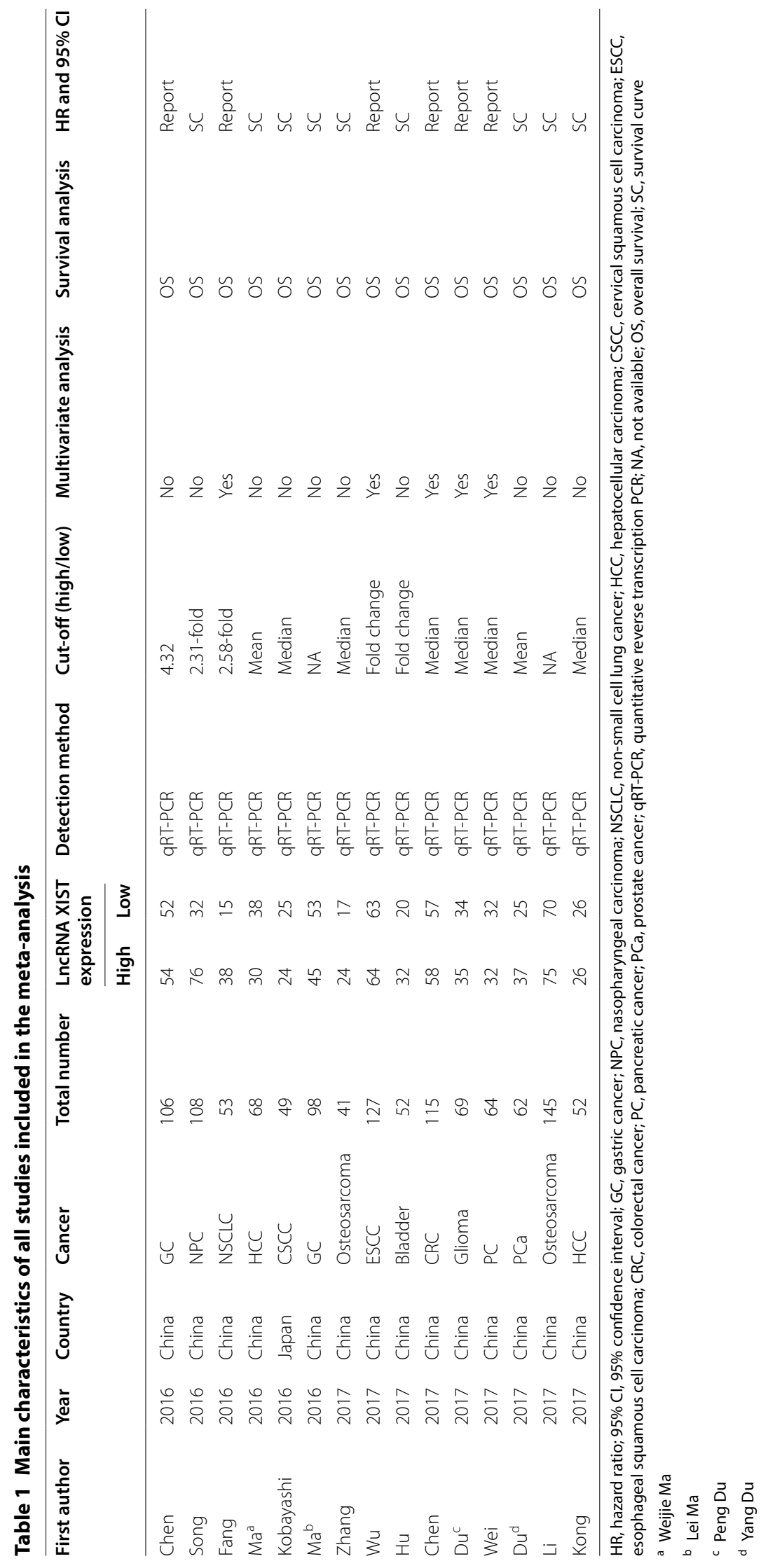


Table 2 The pooled associations between LncRNA XIST expression and the prognosis of solid tumors

\begin{tabular}{|c|c|c|c|c|c|c|}
\hline \multirow[t]{2}{*}{ Outcome subgroup } & \multirow[t]{2}{*}{ Number of patients } & \multirow[t]{2}{*}{ Number of studies } & \multicolumn{2}{|c|}{ Random-effect model } & \multicolumn{2}{|c|}{ Heterogeneity } \\
\hline & & & HR $(95 \% \mathrm{Cl})$ & $p$ value & $I^{2}(\%)$ & $p$ value \\
\hline Overall survival & 1209 & 15 & $1.54(1.07,2.23)$ & 0.021 & 86.3 & $<0.001$ \\
\hline \multicolumn{7}{|l|}{ Tumor type } \\
\hline Digestive system & 630 & 7 & $1.67(1.11,2.51)$ & 0.014 & 80.5 & $<0.001$ \\
\hline Non-digestive system & 579 & 8 & $1.39(0.71,2.74)$ & 0.339 & 90.1 & $<0.001$ \\
\hline \multicolumn{7}{|l|}{ Patients' number } \\
\hline$>65$ & 836 & 8 & $1.75(1.24,2.47)$ & 0.001 & 78.2 & $<0.001$ \\
\hline$\leq 65$ & 373 & 7 & $1.26(0.53,3.01)$ & 0.598 & 91.4 & $<0.001$ \\
\hline \multicolumn{7}{|l|}{ Analysis type } \\
\hline Univariate analysis & 781 & 10 & $1.21(0.75,1.95)$ & 0.436 & 84.8 & $<0.001$ \\
\hline Multivariate analysis & 428 & 5 & $2.39(1.28,4.46)$ & 0.006 & 89.2 & $<0.001$ \\
\hline \multicolumn{7}{|l|}{ HR obtained method } \\
\hline Reported in text & 534 & 6 & $2.50(1.49,4.18)$ & 0.000 & 87.5 & $<0.001$ \\
\hline Data extrapolated & 675 & 9 & $1.07(0.66,1.76)$ & 0.774 & 82.2 & $<0.001$ \\
\hline
\end{tabular}

HR, hazard ratio; 95\% Cl, 95\% confidence interval; OS, overall survival

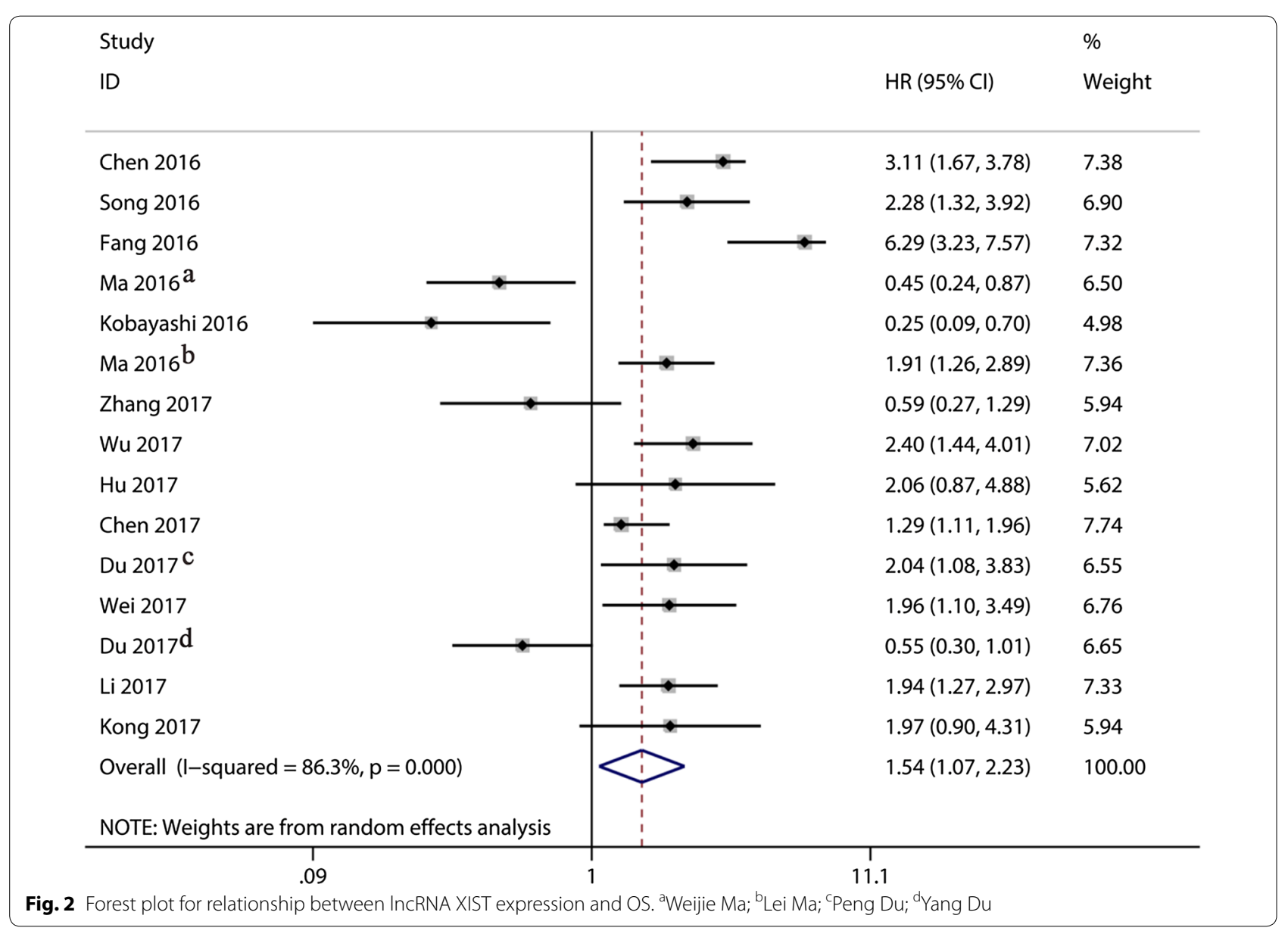


tumor type, patients' number, analysis type, and HR obtained method.

In the subgroup of tumor types, a few studies such as NPC, NSCLC, bladder cancer, glioma, PCa, and osteosarcoma were collectively classified as other tumors for analysis, while more studies on the digestive system alone as a type of analysis. The OS of tumor patients with high expression of lncRNA XIST in digestive system tumors was lower than that in low expression group $(\mathrm{HR}=1.67$, 95\% CI 1.11-2.51, $p=0.014$ ), but not statistically significant in other tumors $(\mathrm{HR}=1.39,95 \% \mathrm{CI} 0.71-2.74$, $p=0.339)$ (Table 2).

Overall, the relationship between high lncRNA XIST expression and prolonged OS was also considered to have statistical significance in other subgroups, including patients' number $>65(\mathrm{HR}=1.75,95 \%$ CI $1.24-2.47$, $p=0.001)$, multivariate analysis $(\mathrm{HR}=2.39,95 \% \mathrm{CI}$ $1.28-4.46, p=0.006)$, reported in text $(\mathrm{HR}=2.50,95 \% \mathrm{CI}$ $1.49-4.18, p=0.000)$, respectively. However, it was not statistically significant in subgroups including patients' number $\leq 65(\mathrm{HR}=1.26,95 \%$ CI $0.53-3.01, p=0.598)$, univariate analysis $(\mathrm{HR}=1.21,95 \%$ CI $0.75-1.95$, $p=0.436)$, and data extrapolated $(\mathrm{HR}=1.07,95 \% \mathrm{CI}$ $0.66-1.76, p=0.774)$. Unfortunately, there was still significant heterogeneity in these studies $\left(\mathrm{I}^{2}>50 \%\right)$ (Table 2$)$.

\section{Sensitivity analysis}

Sensitivity analysis was performed to evaluate the results of meta-analysis results. No significant change was found in the results when any 1 study was excluded, confirming the robustness and reliability of meta-analysis results (Fig. 3).

\section{Publication bias}

A funnel plot, with regard to the publication bias of all studies, showed the basic symmetrical. All $p<0.05$ (twosided) were considered as significant. Begg's test suggested the publication bias was not significant (Begg's Test $p=0.233$ ) (Fig. 4).

\section{Discussion}

LncRNA XIST was a product of the XIST gene located in the $\mathrm{X}$ inactivation center [14], which was the first regulatory RNAs discovered to be involved in the formation of the inactive X chromosome [19]. When an X chromosome was inactivated in female animal, lncRNA XIST diffuses throughout the $\mathrm{X}$ chromosome, ultimately resulting in inactivation of the $\mathrm{X}$ chromosome [20]. Moreover, lncRNA XIST could play a dosage compensation role in female animal cells. In other words, the phenotypes determined by the gene on the $\mathrm{X}$ chromosome were equally expressed in XY males and XX females [15].

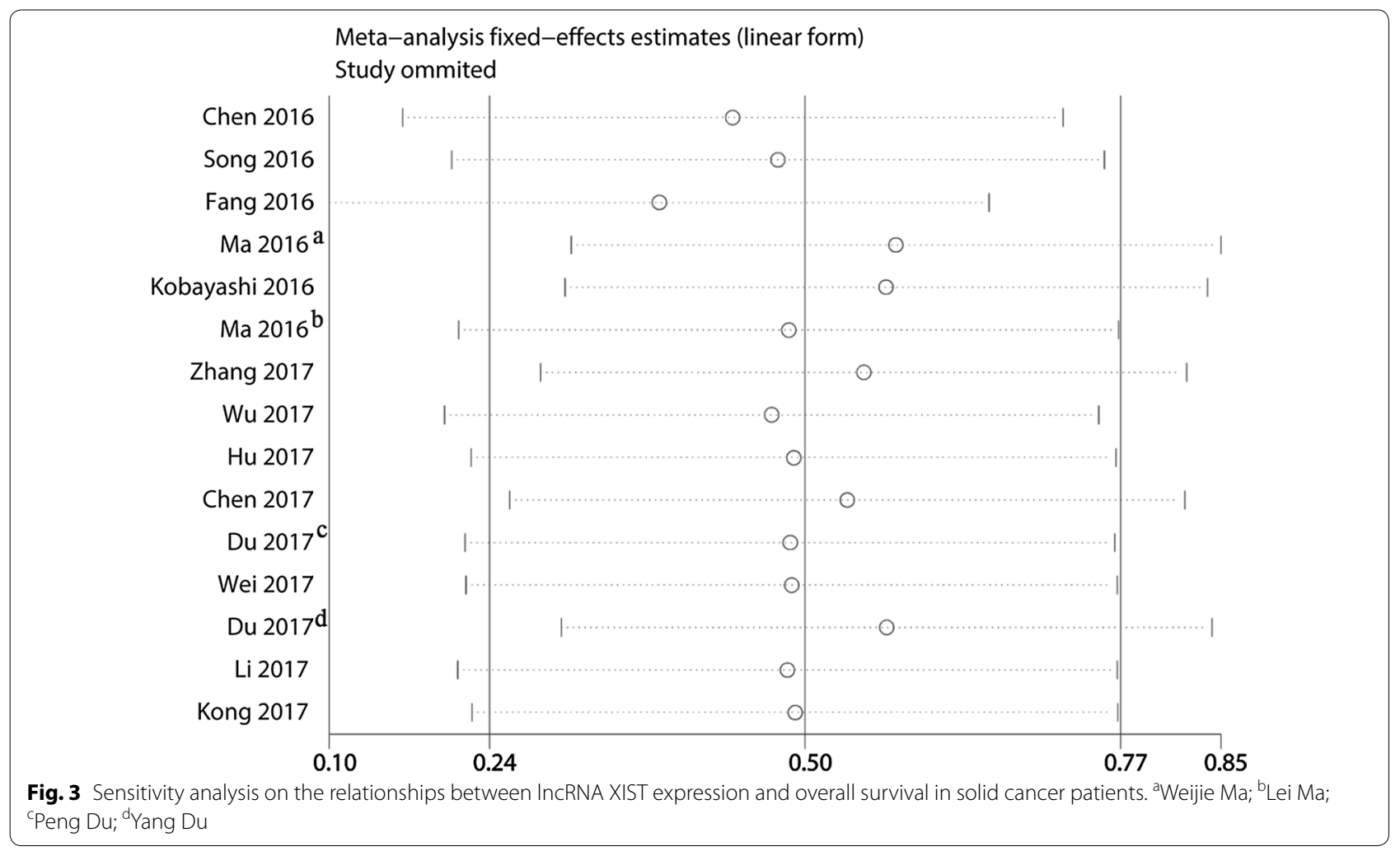




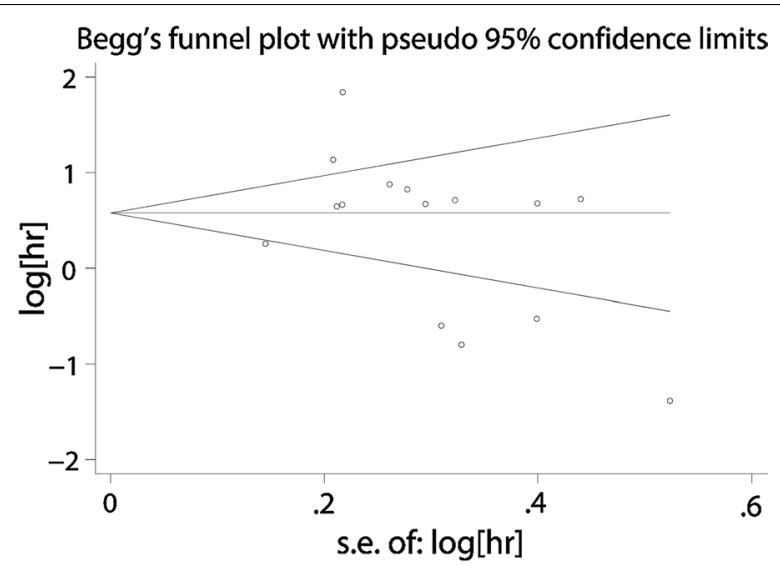

Fig. 4 Funnel plots of publication biases on the relationships between IncRNA XIST expression and overall survival in solid cancer patients

Aberrant expression of lncRNA XIST has been detected in many diseases. It played an important role in proliferation, migration and invasion in cancer cells in vitro and in vivo, which indicated that XIST exerted an essential role on the occurrence and development of various tumors. Differentially expressed lncRNAs could act as oncogenes or tumor suppressors to improve cancer diagnosis, discover potential treatment targets, and improve prognosis. Although many studies found that the high expression of lncRNA XIST was closely related to the prognosis of a variety of tumors, the results of the studies were quite different. It was reported that the high expression of lncRNA XIST was a risk factor for the prognosis of cancers, while some reports indicated that the high expression of lncRNA XIST was a beneficial factor in the prognosis of cancers.

For instance, Chen et al. and $\mathrm{Wu}$ et al. demonstrated that knockdown of lncRNA XIST suppressed cells proliferation, migration and invasion in vitro as well as tumorigenesis and metastasis in vivo in GC (2016) and ESCC (2017), respectively. Moreover, they all found that an inverse relationship between lncRNA XIST and miR-101, and knockdown of lncRNA XIST exerted its tumor-suppressive effects at least in part through regulating miR101 to modulate EZH2 expression [14, 21]. Meanwhile, a study from $\mathrm{Ma}$ et al. showed that IncRNA XIST promoted cell cycle progression from the G1 phase to the S phase and protected cells from apoptosis, which contributed to GC cell growth. XIST was responsible for GC cell proliferation and invasion through the miR-497/MACC1 axis [13]. Furthermore, Temozolomide (TMZ) was the most commonly used alkylating agent in glioma chemotherapy. The data from $\mathrm{Du}$ et al. revealed that XIST knockdown could sensitize TMZ-resistant glioma cells to
TMZ. XIST inhibited miR-29c expression by direct targeting in TMZ-resistant glioma cells [11]. In summary, it implicated that overexpression of lncRNA XIST was associated with adverse prognosis and could be used as an independent prognostic factor.

Contrary to the above tumors, increasing evidence demonstrated that XIST could also act as tumor suppressors, and played important roles in the initiation and progression of multiple cancers [12, 15, 22, 23]. For example, Kobayashi et al. observed in 2016 that the 4-year overall survival rates of patients with CSCC were 87.1 and $54.4 \%$ in the high and low XIST expression groups, respectively [22]. The results suggest that XIST could be a potential biomarker or therapeutic target for OS. However, the effect aberrant XIST expression on the prognosis of patients was still controversial in HCC and osteosarcoma. Recently, a study from Ma et al. showed that patients with JPX/XIST overexpression in HCC had longer survival times than those with low expression [15], contrary to previous research from Kong et al. [16]. Furthermore, a study from Zhang et al. revealed that lncRNA XIST regulated PDCD4 expression by interacting with miR-21-5p and inhibits osteosarcoma cell growth and metastasis [23]. While a study from Li et al. suggested that lncRNA XIST had a tumor promoter effect, and thus, to be a predictor of outcome in patients with osteosarcoma [24].

To get more accurate evidence to prove the relationship between the high expression of lncRNA XIST and the prognosis of cancers, relevant studies have been comprehensively retrieved and analyzed. Furthermore, the regulatory mechanism involved in lncRNA XIST was complex. And there was a lack of systematic research for effect of lncRNA XIST expression on tumor prognosis. Therefore, we conducted a meta-analysis to evaluate the potential value of lncRNA XIST as a novel biomarker for predicting tumor prognosis, which provided a reference for the follow-up study.

In this study, high expression of lncRNA XIST in cancer tissue was associated with poor prognosis in cancer patients $(\mathrm{HR}=1.54,95 \% \mathrm{CI} 1.07-2.23, p=0.021)$, with heterogeneity in the data $\left(\mathrm{I}^{2}>50 \%\right)$. Numerous studies have shown that lncRNAs were involved in the regulation of protein-coding genes at the transcriptional and posttranscriptional levels, and it also influenced the signaling pathway pathways both intracellular as well as in organism development, thus affecting cell growth, apoptosis, and metastasis. Based on the above, deregulations of lncRNA could be a major cause of disease in humancomplex diseases, including tumors. It indicated that it might serve as a negative prognostic marker for solid tumors.

Subgroup analysis and sensitivity analysis were used to investigate whether the heterogeneity of the data affected 
the interpretation of the analysis results. The association between IncRNA XIST overexpression and worse OS was statistically significant in digestive system tumors ( $\mathrm{HR}=1.67,95 \%$ CI 1.11-2.51, $p=0.014$, random-effect). These results indicated that the adverse prognostic effect of high lncRNA XIST remained substantial in digestive system cancers. In the meantime, lncRNA XIST overexpression was associated with a poor prognosis, which was statistically significant when the patients' number is greater than 65 [patients' number $>65(\mathrm{HR}=1.75$, 95\% CI 1.24-2.47, $p=0.001$, random-effect)], multivariate analysis $(\mathrm{HR}=2.39,95 \% \mathrm{CI} 1.28-4.46, p=0.006$, random-effect), and reported in text $(\mathrm{HR}=2.50,95 \% \mathrm{CI}$ $1.49-4.18, p=0.000$, random-effect).

However, there were several limitations in this paper. First of all, the different thresholds of lncRNA XIST expression were different in different studies that could not reach a uniform standard. Second, HR and 95\% CI in some studies could not be obtained directly from the original literature, but HR estimates were derived from their survival curves, which might affect the results of this study. Furthermore, the limited number of included studies, all from Asians, and the small sample size (1290 cases in total) might diminish the reliability of the results. In the future, the studies of high-quality samples needed to be further confirmed.

\section{Conclusion}

In conclusion, the high expression of lncRNA XIST was a close associate to the poor prognosis of cancer patients. LncRNA XIST overexpression might be a new unfavorable prognostic biomarker helpful for the clinical decision-making process. Considering the limitations of this analysis, this conclusion should be viewed with caution. In the future, larger sample sizes were require to confirm the prognostic value of IncRNA XIST in cancer patients and to explore more effective treatment strategies.

\section{Abbreviations \\ HR: hazard ratio; 95\% Cl: 95\% confidence interval; GC: gastric cancer; NPC: nasopharyngeal carcinoma; NSCLC: non-small cell lung cancer; HCC: hepato- cellular carcinoma; CSCC: cervical squamous cell carcinoma; ESCC: esophageal squamous cell carcinoma; CRC: colorectal cancer; PC: pancreatic cancer; PCa: prostate cancer; qRT-PCR: quantitative reverse transcription PCR; NA: not avail- able; OS: overall survival; SC: survival curve. \\ Authors' contributions \\ HM collected and analyzed the data, wrote the paper; KW analyzed the data; YF and JZ conceived and designed this study, analyzed the data; and all authors reviewed the paper. All authors read and approved the final manuscript.}

\section{Author details}

${ }^{1}$ Comprehensive Laboratory, Changzhou Key Lab of Individualized Diagnosis and Treatment Associated with High Technology Research, The Third Affiliated Hospital of Soochow University, Changzhou 213003, China. ${ }^{2}$ Department of Urology, The Third Affiliated Hospital of Soochow University, Changzhou 213003, China.

\section{Acknowledgements}

We would like to thank the researchers and study participants for their contributions.

\section{Competing interests}

The authors declare that they have no competing interests.

\section{Availability of data and materials}

All data generated or analysed during this study are included in this published article.

\section{Consent for publication}

Consent for publication was obtained from the participants.

\section{Ethics approval and consent to participate}

All procedures followed were in accordance with the ethical standards of the responsible committee on human experimentation (the Institutional Ethics Committee of the Third Affiliated Hospital of Soochow University) and with the Helsinki Declaration of 1964 and later versions.

\section{Funding}

This work was supported by the Changzhou High-Level Medical Talents Training Project (No: 2016ZCL002).

\section{Publisher's Note}

Springer Nature remains neutral with regard to jurisdictional claims in published maps and institutional affiliations.

Received: 15 January 2018 Accepted: 6 March 2018

Published online: 09 March 2018

\section{References}

1. Pontier DB, Gribnau J. Xist regulation and function eXplored. Hum Genet. 2011;130:223-36.

2. Mira-Bontenbal H, Gribnau J. New Xist-interacting proteins in X-chromosome inactivation. Curr Biol. 2016;26:338-42.

3. Salvador MA, Wicinski J, Cabaud O, Toiron Y, Finetti P, Josselin E, Lelievre $H$, Kraus-Berthier L, Depil S, Bertucci F. The histone deacetylase inhibitor abexinostat induces cancer stem cells differentiation in breast cancer with low Xist expression. Clin Cancer Res. 2013;19:6520-31.

4. Hu Y, Deng C, Zhang H, Zhang J, Peng B, Hu C. Long non-coding RNA XIST promotes cell growth and metastasis through regulating miR$139-5 p$ mediated Wnt/ $\beta$-catenin signaling pathway in bladder cancer. Oncotarget. 2017;8(55):94554-68.

5. Song P, Ye LF, Zhang C, Peng T, Zhou XH. Long non-coding RNA XIST exerts oncogenic functions in human nasopharyngeal carcinoma by targeting miR-34a-5p. Gene. 2016;592:8-14.

6. Wei W, Liu Y, Lu Y, Yang B, Tang L. LncRNA XIST promotes pancreatic cancer proliferation through miR-133a/EGFR. J Cell Biochem. 2017:118(10):3349-58.

7. Song H, He P, Shao T, Li Y, Li J, Zhang Y. Long non-coding RNA XIST functions as an oncogene in human colorectal cancer by targeting miR132-3p. J BU ON. 2017;22(3):696-703.

8. Xiao Y, Yurievich UA, Yosypovych SV. Long noncoding RNA XIST is a prognostic factor in colorectal cancer and inhibits 5-fluorouracil-induced cell cytotoxicity through promoting thymidylate synthase expression. Oncotarget. 2017;8(47):83171-82.

9. Chen DL, Chen LZ, Lu YX, Zhang DS, Zeng ZL, Pan ZZ, Huang P, Wang FH, $\mathrm{Li} Y \mathrm{YH}$, Ju HQ, Xu RH. Long noncoding RNA XIST expedites metastasis and modulates epithelial-mesenchymal transition in colorectal cancer. Cell Death Dis. 2017:8:e3011.

10. Yao Y, Ma J, Xue Y, Wang P, Li Z, Liu J, Chen L, Xi Z, Teng H, Wang Z. Knockdown of long non-coding RNA XIST exerts tumor-suppressive functions 
in human glioblastoma stem cells by up-regulating miR-152. Cancer Lett. 2015;359:75-86.

11. Du P, Zhao H, Peng R, Liu Q, Yuan J, Peng G, Liao Y. LncRNA-XIST interacts with miR-29c to modulate the chemoresistance of glioma cell to TMZ through DNA mismatch repair pathway. Biosci Rep. 2017;37(5):BSR20170696.

12. Du Y, Weng XD, Wang L, Liu XH, Zhu HC, Guo J, Ning JZ, Xiao CC. LncRNA XIST acts as a tumor suppressor in prostate cancer through sponging miR-23a to modulate RKIP expression. Oncotarget. 2017;8(55):94358-70.

13. Ma L, Zhou Y, Luo X, Gao H, Deng X, Jiang Y. Long non-coding RNA XIST promotes cell growth and invasion through regulating miR-497/MACC1 axis in gastric cancer. Oncotarget. 2017;8(3):4125-35.

14. Chen DL, Ju HQ, Lu YX, Chen LZ, Zeng ZL, Zhang DS, Luo HY, Wang F, Qiu MZ, Wang DS. Long non-coding RNA XIST regulates gastric cancer progression by acting as a molecular sponge of miR-101 to modulate EZH2 expression. J Exp Clin Cancer Res. 2016;35(1):142.

15. Ma W, Wang H, Jing W, Zhou F, Chang L, Hong Z, Liu H, Liu Z, Yuan Y. Downregulation of long non-coding RNAs JPX and XIST is associated with the prognosis of hepatocellular carcinoma. Clin Res Hepatol Gastroenterol. 2017:41:163-70.

16. Kong Q, Zhang S, Liang C, Zhang Y, Kong Q, Chen S, Qin J, Jin Y. LncRNA XIST functions as a molecular sponge of miR-194-5p to regulate MAPK1 expression in hepatocellular carcinoma cell. J Cell Biochem. 2017. https:// doi.org/10.1002/jcb.26540.

17. Tantai J, Hu D, Yang Y, Geng J. Combined identification of long noncoding RNA XIST and HIF1A-AS1 in serum as an effective screening for non-small cell lung cancer. Int J Clin Exp Pathol. 2015;8(7):7887-95.
18. Fang J, Sun CC, Gong C. Long noncoding RNA XIST acts as an oncogene in non-small cell lung cancer by epigenetically repressing KLF2 expression. Biochem Biophys Res Commun. 2016;478:811-7.

19. Monfort A, Wutz A. Progress in understanding the molecular mechanism of Xist RNA function through genetics. Philos Trans R Soc Lond B Biol Sci. 2017;372(1733):20160368.

20. Lu Z, Carter AC, Chang HY. Mechanistic insights in X-chromosome inactivation. Philos Trans R Soc Lond B Biol Sci. 2017;372(1733):20160356.

21. Wu X, Dinglin X, Wang X, Luo W, Shen Q, Li Y, Gu L, Zhou Q, Zhu H, Li Y. Long noncoding RNA XIST promotes malignancies of esophageal squamous cell carcinoma via regulation of miR-101/EZH2. Oncotarget. 2017;8(44):76015-28.

22. Kobayashi R, Miyagawa R, Yamashita H, Morikawa T, Okuma K, Fukayama M, Ohtomo K, Nakagawa K. Increased expression of long non-coding RNA XIST predicts favorable prognosis of cervical squamous cell carcinoma subsequent to definitive chemoradiation therapy. Oncol Lett. 2016;12:3066-74.

23. Zhang R, Xia T. Long non-coding RNA XIST regulates PDCD4 expression by interacting with MIR-21-5p and inhibits osteosarcoma cell growth and metastasis. Int J Oncol. 2017;51:1460-70.

24. Li GL, WU YX, Li YM, Li J. High expression of long non-coding RNA XIST in osteosarcoma is associated with cell proliferation and poor prognosis. Eur Rev Med Pharmacol Sci. 2017;21(12):2829-34.

\section{Submit your next manuscript to BioMed Central and we will help you at every step:}

- We accept pre-submission inquiries

- Our selector tool helps you to find the most relevant journal

- We provide round the clock customer support

- Convenient online submission

- Thorough peer review

- Inclusion in PubMed and all major indexing services

- Maximum visibility for your research

Submit your manuscript at www.biomedcentral.com/submit
() Biomed Central 